\title{
Lernentwicklungsgespräche und die Entwicklung motivationaler Aspekte des Lernens
}

\author{
Sonja Ertl $(\mathbb{D} \cdot$ Benjamin Kücherer $\mathbb{D}$ - Andreas Hartinger $(\mathbb{D}$
}

Eingegangen: 30. November 2020 / Angenommen: 29. März 2021 / Online publiziert: 20. Januar 2022 (C) Der/die Autor(en) 2022

Zusammenfassung Lernentwicklungsgespräche (LEG) sind eine Form der Leistungsbeurteilung und -rückmeldung, in der Elemente Formativen Assessments umgesetzt werden können. Der Beitrag geht der Frage nach, inwieweit die Qualität von LEG (operationalisiert über die Einschätzung der Schüler*innen) mit der Entwicklung motivationaler Aspekte des Lernens (Anstrengungsbereitschaft und schulisches Selbstkonzept) zusammenhängt. Dazu wurden in einem Prä-Post-Design 392 Kinder der Jahrgangsstufe 2 befragt. Es zeigte sich, dass aus Sicht der Kinder die LEG überwiegend positiv eingeschätzt werden - allerdings mit deutlichen Unterschieden. Die Ergebnisse belegen, dass es Zusammenhänge von LEG mit motivationalen Aspekten des Lernens gibt - in Abhängigkeit von der Umsetzung aus der Sicht der Kinder. Die Ergebnisse werden diskutiert und weitere Forschungsdesiderata aufgezeigt.

Schlüsselwörter Lernentwicklungsgespräche $\cdot$ Schüler-Lehrer-Eltern-Gespräche · Formatives Assessment · Motivation

\footnotetext{
Prof. Dr. Sonja Ertl $(\bowtie)$

Institut für Grundschulforschung, Friedrich-Alexander-Universität Erlangen-Nürnberg, Regensburger Straße 160, 90478 Nürnberg, Deutschland

E-Mail: sonja.ertl@fau.de

Benjamin Kücherer · Prof. Dr. Andreas Hartinger

Lehrstuhl Grundschulpädagogik und Grundschuldidaktik, Universität Augsburg,

Universitätsstraße 10, 86159 Augsburg, Deutschland

Benjamin Kücherer

E-Mail: benjamin.kuecherer@phil.uni-augsburg.de

Prof. Dr. Andreas Hartinger

E-Mail: andreas.hartinger@phil.uni-augsburg.de
} 


\title{
Student-teacher-conferences and the development of motivational aspects of learning
}

\begin{abstract}
Student-Teacher-Conferences (German: Lernentwicklungsgespräche= LEG) are a form of assessment and feedback in which elements of Formative Assessment can be implemented. This article examines whether the quality of LEGs (operationalized through student perceptions) correlates with the development of motivational aspects of learning (effort readiness and academic self-concept). For this purpose, 392 children in grade 2 were interviewed in a pre-post design. The results show that, from the children's point of view, LEGs are mostly implemented in a good way-with significant differences. In addition, LEG are connected with motivational aspects of learning-depending on the implementation from the children's perspective. The results will be discussed and further research desiderata identified.
\end{abstract}

Keywords Lernentwicklungsgespräche $\cdot$ Student-Teacher-Conferences · Formative assessment $\cdot$ Motivation

\section{Problemaufriss}

Leistungsbeurteilung, verstanden als Einordnung einer festgestellten Leistung in einen Vergleichsmaßstab, soll (neben einigen gesellschaftlichen und bildungspolitischen Funktionen) vor allem die Förderung weiterer Lernprozesse unterstützen (Worbach et al. 2019, S. 506; Sacher 2014, S. 22f.) - sowohl auf inhaltlicher als auch auf motivationaler Ebene. Dass von Leistungsbeurteilungen und den damit verbundenen Leistungsrückmeldungen positive Effekte auf Leistung und Motivation ausgehen können, zeigen zahlreiche Befunde aus der Forschung zu Feedback und zum Formativen Assessment (z. B. Furtak et al. 2016; Harks et al. 2014; Hattie 2009; Maier 2010).

Bezogen auf die Rückmeldung ist dabei sowohl die Form als auch die Qualität der Umsetzung von Bedeutung. Lange bekannt ist, dass es nicht möglich ist, ausschließlich durch Ziffernnoten eine informative Rückmeldung (im Sinne eines lernförderlichen Feedbacks) zu geben (vgl. dazu schon Ingenkamp 1971). Verbalbeurteilungen haben hier bessere Möglichkeiten; allerdings zeigte sich in Studien keine Überlegenheit gegenüber Ziffernnoten (z.B. Valtin und Wagner 2002). Dies lässt sich durch die Qualität der Verbalbeurteilungen erklären: Weitgehend stellten sie eine informationsarme Übersetzung von Noten in Textform dar (Schmude 2002; Sacher 2014). Damit unterschieden sie sich zwar in der Form von Ziffernnoten, nicht jedoch in ihrem Informationsgehalt.

Lernentwicklungsgespräche (LEG) haben das Potenzial, Leistungsbeurteilung und die anschließende Leistungsrückmeldung so umzusetzen, dass günstige motivationale Aspekte des Lernens unterstützt werden. Denn sie berücksichtigen in ihrer Konzeption verschiedene Elemente des Formativen Assessments, wie z. B. die Selbsteinschätzung der Lernenden, informative Rückmeldungen, die für das weitere Lernen hilfreich sind, und die Vereinbarung klarer Ziele, deren Erreichen im wei- 
teren Schuljahr überprüft werden soll (Dollinger et al. 2020a). Zu untersuchen ist jedoch, in welcher Qualität diese Elemente umgesetzt werden und inwieweit dadurch Auswirkungen auf die Motivation und das Lernen der Schüler*innen erreicht werden können.

\section{Lernentwicklungsgespräche}

Lernentwicklungsgespräche sind Gespräche zwischen einer Lehrperson und einem Kind im Beisein mindestens eines Erziehungsberechtigten über Lernstand, Lernprozess und (die vergangene und künftige) Lernentwicklung des Kindes (Betz et al. 2019; Bonanati 2018; Wilhelm 2015; Dollinger et al. 2020b). In dem 15- bis 30minütigen Gespräch, in dem auch die Selbsteinschätzung der Kinder berücksichtigt werden soll, sollen Stärken und Schwächen sowie das Entwicklungspotenzial des jeweiligen Kindes im Mittelpunkt stehen - auch durch die Planung konkreter Schritte für das weitere Lernen und die Vereinbarung entsprechender Ziele (z. B. Bonanati 2018; Wilhelm 2015). Dabei haben LEG in unterschiedlichen Bundesländern eine unterschiedliche Bedeutung und Verankerung. So können z. B. in Bayern LEG das Zwischenzeugnis und in den Jahrgangsstufen 1 und 3 auch das Jahreszeugnis ersetzen. In anderen Bundesländern, wie z. B. in Hamburg und Thüringen, werden die Gespräche zusätzlich zu den Zeugnissen mindestens einmal pro Schuljahr geführt. Damit betrachten LEG die Lernentwicklung über einen längeren Zeitraum, wie beispielsweise ein Schulhalbjahr.

Konkrete Vorgaben zur Durchführung beziehen sich in aller Regel lediglich auf organisatorische Aspekte, wie z.B. den Teilnehmendenkreis, die Dokumentationspflicht, den Zeitraum der Durchführung oder allgemeine Inhalte wie Stärken und Schwächen (z. B. Dollinger et al. 2020b). Dennoch lassen sich Elemente finden, die für gewöhnlich in LEG umgesetzt werden (z. B. Häbig 2018; ebd.): So füllen die Kinder in aller Regel vor dem Gespräch einen Selbsteinschätzungsbogen oder ein Reflexionsinstrument (wie z. B. eine Lernlandkarte o. Ä.; Nett und Schilling 2020) aus und halten darin die eigene Wahrnehmung ihrer Kompetenzen fest; die Lehrpersonen schätzen ebenfalls im Vorfeld die Kompetenzen der Lernenden ein - der dazu verwendete Bogen dient dann normalerweise als Dokumentationsgrundlage im Gespräch. Im Gespräch selbst erhalten die Lernenden Rückmeldung zu ihren Lernleistungen und planen gemeinsam mit der Lehrperson ihr weiteres Lernen, indem für gewöhnlich Ziele vereinbart werden, die dann im weiteren Verlauf des Schuljahres überprüft werden sollen.

Bzgl. der Frage, wann LEG qualitätvoll, lernförderlich gestaltet sind, lohnt ein Blick auf die Befundlage zu Feedback (z. B. Harks et al. 2014) und Formativem Assessment (z. B. Schmidinger et al. 2016; Black und Wiliam 2009). Aus dieser lassen sich Qualitätskriterien für die Vorbereitung und Durchführung der LEG sowie die Weiterarbeit mit den vereinbarten Zielen ableiten (Dollinger et al. 2020a; Dollinger und Hartinger 2019).

Drei der dort benannten Kriterien, die sich auf die konkrete Gesprächssituation beziehen und für die motivationale Entwicklung der Lernenden von Bedeutung sind, sollen hier näher beachtet werden: a) die Berücksichtigung der Selbsteinschät- 
zung des Kindes, b) lernunterstützende Rückmeldungen sowie c) die Vereinbarung passender, hilfreicher Ziele für das weitere Lernen.

Wird im LEG die Selbsteinschätzung des Kindes berücksichtigt, so ist diese Selbsteinschätzung im Gespräch nicht nur konkret zu erfragen. Zusätzlich ist sie aufzunehmen - auch in einen Austausch, wenn es unterschiedliche Sichtweisen gibt. Dass eine solche Berücksichtigung der Selbsteinschätzung der Lernenden von Bedeutung ist, zeigen Befunde mit positiven Effekten von Selbstbewertung/ Selbstreflexion bzw. self-assessment auf Motivation, Selbstwirksamkeit und Leistung (zsfd. Andrade 2010; Schunk 1996).

Unter dem Kriterium der lernunterstützenden Rückmeldung (z. B. Hattie und Timperley 2007; Kollar und Fischer 2019; Harks et al. 2014) wird zunächst gefasst, dass Informationen zum Lernstand und zur Lernentwicklung gegeben werden. Gerade die Berücksichtigung der Lernentwicklung, und damit der Bezug zur individuellen Bezugsnorm, lässt erwarten, dass die Anstrengung als lernförderliche Attribution in den Fokus rückt, sowie dass Schwächen, die im sozialen Vergleich deutlich werden, weniger dominant in Erscheinung treten. Zudem gelten Strategien zur Unterstützung des weiteren Lernprozesses - und damit die Unterstützung des selbstständigen Lernens - als wichtiges Element einer lernförderlichen Rückmeldung (zsfd. Kollar und Fischer 2019).

Die Vereinbarung passender, hilfreicher Ziele gilt ebenfalls als wichtiges Kriterium eines gelungenen Feedbackprozesses, der den Blick nicht nur zurück, sondern auch nach vorne (als Feed-Forward; z. B. Hattie und Timperley 2007) richtet. Als günstig gilt, wenn die Lernenden die vereinbarten Ziele inhaltlich und bezogen auf die Schwierigkeit als adäquat einschätzen, wenn sie motiviert sind, diese Ziele zu erreichen und auch wissen, wie sie ihre Ziele erreichen können (zsfd. z. B. Brandstätter und Hennecke 2018).

$\mathrm{Zu}$ LEG und ihren Auswirkungen auf schulisches Lernen existieren bislang noch kaum empirische Befunde. Bonanati (2018) und Mundwiler (2017) untersuchten LEG aus gesprächsanalytischer Perspektive mit dem Fokus auf Partizipations- und Gesprächsstrukturen. Im Mittelpunkt der Studie von Häbig (2018) an Gymnasien stand die Prozessqualität von LEG aus Sicht der Lernenden. Eigene (Vor-)Untersuchungen zeigten, dass die Rückmeldung im LEG von allen Beteiligten sowie aus externer Beobachtersicht weitestgehend als lernunterstützend eingeschätzt wird, wenngleich die Umsetzung der einzelnen Elemente dabei erheblich variiert (Dollinger 2019, 2020; Ertl et al. im Druck). Des Weiteren liegen erste Befunde vor, die Hinweise darauf geben, dass die Umsetzung der Qualitätskriterien einer großen Varianz unterliegt (Dollinger und Hartinger 2019), die sich auch bei Betz et al. (2019, S. $67 \mathrm{ff}$.) widerspiegelt.

Uns sind jedoch keine Studien bekannt, die Lernentwicklungsgespräche in Verbindung mit dem nachfolgenden Lernen der Kinder untersuchen. Die vorliegende Studie möchte einen Beitrag zur Bearbeitung dieses Forschungsdesiderats leisten. Im Fokus stehen dabei mit der Anstrengungsbereitschaft und dem schulischen Selbstkonzept zwei für den Lernerfolg zentrale motivationale Aspekte des Lernens (zsfd. z. B. Grassinger et al. 2019). 


\section{Motivationale Aspekte des Lernens}

\subsection{Anstrengungsbereitschaft}

Unter Anstrengung wird allgemein ,die Mobilisierung von energetischen Ressourcen, die notwendig sind, um eine Tätigkeit auszuführen“, verstanden (Rollet und Rollet 2018, S. 8). Die Bereitschaft, sich für schulische Lernprozesse anzustrengen, ist dabei ein zentraler Teil der schulischen Lern- und Leistungsmotivation. Als Merkmal der Person wird sie im Rahmenmodell der Lern- und Leistungsmotivation (Dresel und Lämmle 2017, S. 86f.) als motivationale Tendenz verortet, die dann in spezifischen Lern-Situationen zur aktuellen Motivation für diese Lernhandlung und damit zu einer erhöhten Anstrengungsquantität führt. Somit ist sie für die Ausführung einer Handlung von großer Bedeutung, auch da die Höhe der Motivation mitentscheidend ist, dass Handlungen überhaupt begonnen werden (Grassinger et al. 2019, S. 209).

Die Bedeutsamkeit der Anstrengungsbereitschaft für schulisches Lernen und den schulischen Lernerfolg ist gut belegt. So konnte z. B. Müller (2013) zeigen, dass die Anstrengungsbereitschaft ein wichtiger Prädiktor für den Schulerfolg zum Ende der Grundschulzeit bis in die Sekundarstufe hinein ist. Zudem wird sie von Lehrpersonen (und auch Eltern) bei der Entscheidung für die Schullaufbahnpräferenz am Ende der Grundschulzeit mitberücksichtigt (Stubbe et al. 2012). Umgekehrt hat Anstrengungsvermeidung von Schüler*innen negative Auswirkungen auf den Lernerfolg - auch unter der Berücksichtigung korrelierender Variablen, wie der Furcht vor Misserfolg (zsfd. Rollet und Rollet 2018, S. 10).

Die Entwicklung von Anstrengungsbereitschaft (oder im umgekehrten Fall der Anstrengungsvermeidung) wird durch verschiedene Aspekte beeinflusst. Gut belegt ist ein negativer Zusammenhang zwischen Anstrengungsvermeidung und der Hoffnung auf Erfolg (Rollet und Rollet 2018). Die Unterstützung dieser Hoffnung auf Erfolg und der damit verbundenen Freude über erreichte Ziele gelten als wichtige Elemente motivationsförderlicher Maßnahmen (zsfd. z. B. Rheinberg und Fries 2018). Von Bedeutung ist dabei auch die Unterstützung lernförderlicher Attributionen auf internal variable Ursachen wie die eigene Anstrengung (zsfd. z. B. Grassinger et al. 2019, S. 221 ff.). Im schulischen Kontext kann dies im Rahmen der Leistungsbeurteilung und -rückmeldung auch dadurch unterstützt werden, indem die individuelle Bezugsnorm (als Vergleich einer Leistung mit vorherigen Leistungen) verwendet wird (z. B. Brunstein und Heckhausen 2018, S. 208). Als wichtiges Merkmal einer motivationsförderlichen Unterrichtsgestaltung gilt auch die Vereinbarung passender Ziele, da Anstrengung durch vorhandene Ziele reguliert wird (Brandstätter und Hennecke 2018, S. 339 ff.). Unter Bezug auf Erwartungs-Wert-Modelle (z. B. Eccles und Wigfield 2002) bedeutet dies, dass diese Ziele von den Schüler*innen selbst sowohl als persönlich bedeutsam als auch als bewältigbar eingeschätzt werden müssen (z. B. Grassinger et al. 2019, S. 221).

Für den schulischen Kontext betont Grittner (2015, S. 165), dass „mit Blick auf die motivationalen Faktoren [.] das interne Feedback wichtig [ist], um mit der Lernaktivität $\mathrm{zu}$ beginnen und sie aufrecht zu erhalten. Dies gelingt insbesondere dann, wenn man sich eigene Stärken und Schwächen vor Augen führt und adaptive 
Bewertungen der Lernergebnisse durchführt“. Somit ist neben dem Aufzeigen der Lernentwicklung und dem Vorhandensein von Zielen auch die Selbsteinschätzung in Zusammenhang mit Anstrengungsbereitschaft wichtig.

Mit Blick auf die oben kurz aufgeführten Erwartungs-Wert-Modelle, ist die Bereitschaft einer Person, sich für eine Aktivität anzustrengen, auch davon abhängig, wie zuversichtlich sie ist, diese bewältigen zu können. Hierbei spielt das Selbstkonzept eine wichtige Rolle.

\subsection{Schulisches Selbstkonzept}

Das Selbstkonzept, verstanden als Vorstellungen einer Person über ihre eigenen Begabungen bzw. Fähigkeiten (z. B. Möller und Trautwein 2015; Moschner und Dickhäuser 2018), ist hierarchisch organisiert und unterteilt sich in ein akademisches (schulisches) Selbstkonzept und ein nicht-akademisches Selbstkonzept mit jeweils weiteren Facetten (Shavelson et al. 1976).

Das schulische Selbstkonzept entwickelt sich im Durchschnitt vom „Optimisten hin zum Realisten“ (Helmke 1998) und scheint sich ab der Grundschulzeit zunehmend zu stabilisieren (ebd.; Praetorius et al. 2016). Hinsichtlich der kausalen Wirkrichtung zwischen Leistung und Selbstkonzept wird von reziproken Effekten ausgegangen, die sich mit dem Self-Enhancement- (Selbstkonzept beeinflusst Leistung) bzw. dem Skill-Development-Ansatz (Leistung beeinflusst Selbstkonzept) erklären lassen (z.B. Möller und Trautwein 2015; Moschner und Dickhäuser 2018). Die Befundlage für den Grundschulbereich ist dabei nicht ganz eindeutig. So liegen Forschungsergebnisse vor, die zeigen, dass zu Beginn der Grundschulzeit eher das Selbstkonzept die Leistungen beeinflusst, sich diese Wirkrichtung im Laufe der Grundschulzeit jedoch in die entgegengesetzte Richtung verändert (im Überblick Renner et al. 2011). Diese Befunde wurden durch eine Studie von Praetorius et al. (2016) für das Fach Mathematik in der ersten Jahrgangsstufe bestätigt. Ehm et al. (2019) stellen solche reziproken Effekte für den Grundschulbereich, ebenso wie eine Veränderung der Wirkrichtung im Laufe der Grundschulzeit, aufgrund ihrer Befunde jedoch in Frage, da unterschiedliche methodische Verfahren zu unterschiedlichen Ergebnissen führten. Unabhängig davon ist ein ausreichend hohes Selbstkonzept der Schüler*innen anzustreben, da ein geringes schulisches Selbstkonzept mit einer geringeren Anstrengungsbereitschaft und Ausdauer bei schulischen Herausforderungen korreliert (zsfd. Moschner und Dickhäuser 2018).

Für die Entwicklung des schulischen Selbstkonzepts sind Rückmeldungen und Vergleichsprozesse von entscheidender Bedeutung (Moschner und Dickhäuser 2018). So zeigten sich für die (Weiter-)Entwicklung des Selbstkonzepts von Grundschüler*innen die Einschätzungen und Rückmeldungen ihrer Eltern (Poloczek et al. 2011) und Lehrpersonen (Spinath 2004) als bedeutsam. Dabei nutzen Lernende gemäß dem Internal/External Frame of Reference Model (I/E Modell; Marsh 1986) verschiedene Bezugsrahmen für die Einschätzung der eigenen Kompetenzen. Wolff et al. (2018) erweiterten dieses Modell (2 I/E), indem sie neben sozialen (mit Mitschüler*innen) und dimensionalen (mit anderen Fächern) auch temporale Vergleiche (mit eigenen früheren Leistungen) berücksichtigen. Dabei konnten sie zeigen, dass alle drei Vergleichsprozesse in der Selbstkonzeptdarstellung berücksichtigt werden, 
wobei die stärksten Effekte durch soziale, mittlere Effekte durch dimensionale und kleinere Effekte durch temporale Vergleiche entstehen (Wolff et al. 2018, 2019). Als Ursache für fehlende temporale Vergleiche bei jüngeren Grundschüler*innen vermuten sie deren vergleichsweise kurze Schulerfahrung (Wolff et al. 2018).

Die Befunde bestärken die Vermutung, dass von LEG ein Einfluss auf die Entwicklung des schulischen Selbstkonzepts ausgehen kann: Zum einen findet eine Rückmeldung einer relevanten Person statt, zum anderen finden Vergleiche statt auch mit der Selbsteinschätzung. Die Tatsache, dass soziale Vergleiche in LEG kaum gezogen werden - dies zeigen die Befunde einer Beobachtungsstudie (Ertl et al. in Vorbereitung) - gibt gerade für Schüler*innen mit vergleichsweise schlechten Leistungen die Möglichkeit, durch dimensionale und insbesondere temporale Vergleiche ein günstigeres schulisches Selbstkonzept zu entwickeln.

Zusammenfassend lässt sich zunächst festhalten, dass Anstrengungsbereitschaft und schulisches Selbstkonzept Auswirkungen auf das Lernen und die Lernergebnisse von Schüler*innen haben. Selbst beeinflusst werden sie durch die Selbstreflexion/ Selbsteinschätzung der Lernenden, durch den Vergleich mit eigenen früheren Leistungen bzw. anderen Kindern und insbesondere auch durch die Rückmeldungen von wichtigen Bezugspersonen. Die Anstrengungsbereitschaft wird zudem durch Ziele mit einer persönlichen Bedeutsamkeit unterstützt. LEG haben aufgrund ihrer Konzeption das Potenzial, Anstrengungsbereitschaft und schulisches Selbstkonzept zu fördern, 1) indem durch den Fokus auf die Lernentwicklung die Anstrengung des Kindes in den Blick genommen wird, 2) indem - vor allem bei leistungsschwächeren Schüler*innen - selbstkonzeptschädliche soziale Vergleiche vermieden werden, 3) indem die Sinnhaftigkeit von Lernen verdeutlicht sowie 4) indem durch die Vereinbarung passender Ziele die Bedeutsamkeit und die Hoffnung auf das Erreichen dieser Ziele gesteigert wird.

Entscheidend dabei ist jedoch nicht nur die „objektive“ Qualität der Rückmeldung und der Zielsetzungen, sondern auch, inwieweit die Lernenden selbst diese als nützlich und hilfreich wahrnehmen (z. B. Harks et al. 2014), weshalb in der vorliegenden Studie die Wahrnehmung der Lernenden als Maß für die Umsetzung der Qualitätskriterien herangezogen wird.

\section{Fragestellungen und Hypothesen}

Im Fokus dieses Beitrags steht die Frage, inwieweit es Zusammenhänge zwischen der Qualität der LEG (wahrgenommen aus der Sicht der Kinder) und der Entwicklung motivationaler Aspekte des Lernens gibt. Daraus ergeben sich die folgenden Fragestellungen:

1. Wie schätzen die Lernenden die LEG ein?

a. Inwieweit werden aus ihrer Sicht 1) die Selbsteinschätzung im Gespräch berücksichtigt, 2) das LEG als lernunterstützend wahrgenommen und 3) die vereinbarten Ziele als passend und hilfreich erachtet?

b. Wie sehr unterscheiden sich diese Einschätzungen der einzelnen Kinder sowie über die verschiedenen Klassen hinweg? 
2. Welche Zusammenhänge gibt es zwischen diesen drei Einschätzungen und der Entwicklung motivationaler Aspekte des Lernens (Anstrengungsbereitschaft und schulisches Selbstkonzept)?

Wir erwarten aufgrund der Befunde aus einer Vorstudie zur lernunterstützenden Rückmeldung (Dollinger 2019, 2020) im Mittel eine eher positive Einschätzung der Lernenden bezüglich der Berücksichtigung ihrer Selbsteinschätzung, der Umsetzung lernunterstützender Rückmeldungen und der Vereinbarung passender und hilfreicher Ziele. Gleichzeitig erwarten wir, dass die Einschätzung der Kinder variiert, da auch die LEG bei einer Lehrkraft unterschiedlich ablaufen (Ertl et al. im Druck).

Bezüglich der Effekte erwarten wir - gemäß den Befunden zum Formativen Assessment (vgl. Abschn. 1) - folgende Zusammenhänge: Eine günstige Einschätzung der Lernenden bzgl. der drei benannten Bereiche sollte positiv mit der Entwicklung von Anstrengungsbereitschaft und schulischem Selbstkonzepts zusammenhängen.

\section{Methodik}

\subsection{Studiendesign}

Die Studie ist in einem längsschnittlichen Prä-Post-Design angelegt. Die Schüler*innen wurden vor Beginn aller LEG zu ihrer Anstrengungsbereitschaft und ihrem schulischen Selbstkonzept befragt. Am Montag nach ihrem eigenen LEG wurden sie erneut zu diesen beiden motivationalen Aspekten des Lernens befragt und zugleich um ihre Einschätzung des LEG gebeten.

\subsection{Stichprobe}

Die Stichprobe setzt sich aus zwei Kohorten in den Schuljahren 2018/19 und 2019/20 zusammen. Insgesamt nahmen 392 Kinder (178 Jungen, 176 Mädchen, 38 o. A.) aus 61 unterschiedlichen Klassen an beiden Messzeitpunkten teil. Alle Kinder besuchten zum Zeitpunkt der Untersuchung die 2. Jahrgangsstufe.

\subsection{Untersuchungsinstrumente}

Die Einschätzung des LEG sowie die Angaben zur Anstrengungsbereitschaft und zum Selbstkonzept erfolgten über eine 4-stufige Likertskala $(0=$ trifft nicht zu bis $3=$ trifft $\mathrm{zu}$ ). Fehlende Werte (bei allen Items $\leq 2 \%$ zu jedem Messzeitpunkt) wurden mithilfe des EM-Algorithmus in SPSS imputiert.

\subsubsection{Wahrgenommene Qualität der LEG}

Die Einschätzung der LEG durch die Lernenden geschah durch einen selbst entwickelten Fragebogen. Dabei kamen drei verschiedene Subskalen zum Einsatz. Die Subskala „Berücksichtigung der Selbsteinschätzung“ ( $\left.\alpha=0,60, r_{i t}=0,39-0,45\right)$ umfasst vier Items (z. B. Im LEG hat meine Lehrerin mich gefragt, was ich gut kann.), 
„lernunterstützende Rückmeldung“ ( $\left.\alpha=0,79, r_{i t}=0,47-0,58\right)$ sechs Items (z. B. Ich weiß jetzt nach dem LEG genau, warum ich mich verbessert oder verschlechtert ha$b e$.) und „passende und hilfreiche Ziele“ $\left(\alpha=0,65, r_{i t}=0,36-0,43\right)$ fünf Items (z. B. Das, was wir ausgemacht haben, wird mir beim Lernen helfen.).

Diese drei Subskalen korrelieren signifikant miteinander. Die Korrelation zwischen „lernunterstützende Rückmeldung“ und „passende und hilfreiche Ziele“ ist mit $r=0,586$ hoch, die Korrelationen zur „Berücksichtigung der Selbsteinschätzung“ liegen im mittleren Bereich $(r=0,236$ bzw. $r=0,284)$. Aus diesem Grund wurde eine konfirmatorische Faktorenanalyse gerechnet, um festzustellen, ob es sinnvoll ist, die drei Skalen getrennt zu betrachten oder ob eine Lösung mit nur einer Skala oder mit zwei Skalen (wobei „lernunterstützende Rückmeldung“ mit ,passende und hilfreiche Ziele" kombiniert wird) die Daten besser abbildet. Der Befund ist eindeutig: Das Modell mit drei Faktoren erreicht gute Kennwerte $(\mathrm{CFI}=0,95$; RMSEA =0,035; $\left.\mathrm{SRMR}=0,049 ; \mathrm{Chi}^{2} / \mathrm{df}=1,48\right)$. Das Ein-Faktoren-Modell fällt dagegen deutlich ab $\left(\mathrm{CFI}=0,83\right.$; RMSEA =0,058; SRMR =0,064; $\left.\mathrm{Chi}^{2} / \mathrm{df}=2,34\right)$, das Zwei-Faktoren-Modell erreicht weitgehend akzeptable Werte $(\mathrm{CFI}=0,93$; RMSEA $=0,041$; $\left.\mathrm{SRMR}=0,053 ; \mathrm{Chi}^{2} / \mathrm{df}=1,48\right)$ - diese sind jedoch durchgängig schlechter als bei der Lösung mit drei Subskalen. Aus diesem Grund wurde mit den drei - auch inhaltlich gut trennbaren - Skalen weitergerechnet.

\subsubsection{Anstrengungsbereitschaft und schulisches Selbstkonzept}

Zur Erfassung der Anstrengungsbereitschaft kam nahezu vollständig die entsprechende Skala des „Fragebogens zur Erfassung emotionaler und sozialer Schulerfahrungen“ (FEESS; Rauer und Schuck 2003, 2004) zum Einsatz, zur Erfassung des schulischen Selbstkonzepts wurde eine Skala von Munser-Kiefer et al. (2016) adaptiert. Die Reliabilitäten beider Skalen liegen im akzeptablen bis guten Bereich (Anstrengungsbereitschaft: 11 Items; $\alpha_{\mathrm{MZP}} 1: 0,71, r_{i t}=0,26-0,48 ; \alpha_{\mathrm{MZP} 2}: 0,76$, $r_{i t}=0,36-0,52$; Beispielitem: Ich gebe schnell auf, wenn ich Probleme habe - invers kodiert; schulisches Selbstkonzept: 10 Items; $\alpha_{\text {MZP } 1}: 0,67, r_{i t}=0,27-0,48 ; \alpha_{\text {MZP } 2}$ : 0,73, $r_{i t}=0,18-0,58$; Beispielitem: Ich kann auch schwierige Aufgaben lösen.).

\subsection{Auswertung}

Als Maß für die Einschätzung der qualitativen Umsetzung der LEG aus Sicht der Lernenden wurden die Mittelwerte der drei Skalen (Berücksichtigung der Selbsteinschätzung, lernunterstützende Rückmeldung, passende und hilfreiche Ziele) berechnet. Um die Varianz zwischen den einzelnen Klassen zu erfassen, wurden Intraklassenkorrelationen berechnet.

Um zu überprüfen, inwieweit die wahrgenommene Qualität der LEG mit der Entwicklung der Anstrengungsbereitschaft und des schulischen Selbstkonzepts zusammenhängt, wurden Regressionsanalysen berechnet. Dies geschah mithilfe des Programms Mplus (Muthén und Muthén 2017) unter Berücksichtigung der Klasse als Clustervariable (type $=$ complex), um der ,geklumpten“ Stichprobe Rechnung zu tragen. Dies ist aufgrund der vorgefundenen Intraklassenkorrelationskoeffizienten (s. Abschn. 6.1) auch sinnvoll. Neben den LEG-Qualitäts-Prädiktoren wurde 
als weitere unabhängige Variable das Geschlecht der Lernenden kontrolliert. In das Modell wurde zudem der jeweilige Prätestwert integriert.

\section{Ergebnisse}

\subsection{Einschätzung der LEG aus Sicht der Lernenden}

Die LEG wurden aus Sicht der Lernenden überwiegend als positiv eingeschätzt. Dies zeigte sich daran, dass die Mittelwerte aller Skalen signifikant über der theoretischen Mitte von 1,5 lagen. So gaben die Kinder an, dass ihre Selbsteinschätzung im Gespräch angemessen berücksichtigt wurde $(M=2,1 ; S D=0,6$; $t(391)=19,92, p<0,001)$, dass sie lernunterstützende Rückmeldungen $(M=2,6$; $S D=0,5 ; t(391)=43,46, p<0,001)$ erhalten haben und dass passende und hilfreiche Ziele für das weitere Lernen vereinbart wurden $(M=2,6 ; S D=0,4 ; t(391)=47,75$, $p<0,001)$.

Die berechneten ICC mit Werten von 0,093 (Berücksichtigung der Selbsteinschätzung), 0,143 (lernunterstützende Rückmeldung) und 0,082 (passende und hilfreiche Ziele) verweisen auf eine gewisse Varianz zwischen den Klassen. Dies deutet auf systematische Unterschiede in der Umsetzung der Gespräche durch die einzelnen Lehrpersonen hin.

Zugleich gibt es deutliche Unterschiede in der Einschätzung der Kinder innerhalb der einzelnen Klassen. Zum Teil - allerdings nur in wenigen Klassen - wird die Spannweite bei einzelnen Subskalen von 0 bis 3 vollständig ausgeschöpft. Damit bestätigen die Ergebnisse unsere Hypothese, dass die Qualität der LEG aus Sicht der Lernenden grundsätzlich als hoch eingeschätzt wird, dass es bzgl. dieser Einschätzung jedoch zum Teil beachtliche Unterschiede zwischen verschiedenen Klassen und verschiedenen Kindern gibt.

\subsection{Zusammenhänge der Einschätzung von LEG mit motivationalen Aspekten des Lernens}

Die in Tab. 1 aufgelisteten Ergebnisse zeigen, dass sich im Mittel nur geringe Veränderungen ergeben - allerdings steigt das schulische Selbstkonzept $(t(391)=5,03$, $p<0,001)$ über die gesamte untersuchte Stichprobe hinweg überzufällig an.

Die zentrale Fragestellung dieser Studie ist, inwieweit Veränderungen bzgl. motivationaler Aspekte des Lernens mit der Einschätzung der LEG aus Sicht der Lernenden zusammenhängen. In Tab. 2 sind die Ergebnisse zusammengefasst. Gezeigt

Tab. 1 Mittelwerte der Skalen zu motivationalen Aspekten des Lernens im Prä- und Post-Test

\begin{tabular}{lll}
\hline & Prä-Test & Post-Test \\
& $M(S D)$ & $M(S D)$ \\
\hline Anstrengungsbereitschaft & $2,58(0,41)$ & $2,55(0,48)$ \\
Schulisches Selbstkonzept*** & $2,06(0,44)$ & $2,16(0,45)$ \\
\hline
\end{tabular}

Zweiseitiger t-Test bei abhängigen Stichproben

$* p \leq 0,05 ; * * p \leq 0,01 ; * * * p \leq 0,001$ 
Tab. 2 Betagewichte der Einschätzung der LEG auf die untersuchten motivationalen Aspekte des Lernens

\begin{tabular}{lll}
\hline & Anstrengungsbereitschaft & Schulisches Selbstkonzept \\
\hline Prätest & $0,394^{* * *}$ & $0,578^{* * *}$ \\
Geschlecht & 0,120 & $0,257^{* *}$ \\
Berücksichtigung der Selbsteinschätzung & $-0,024$ & $-0,050$ \\
Lernunterstützende Rückmeldung & $0,460^{*}$ & $0,378^{* *}$ \\
Passende und hilfreiche Ziele & $0,412^{*}$ & 0,103 \\
$\mathrm{R}^{2}$ & 0,342 & 0,408 \\
\hline
\end{tabular}

Jeweils einseitig getestet; Geschlecht: $0=$ Mädchen, $1=$ Jungen

$R^{2}$ erklärte Stichprobenvarianz

$* p<0,05 ; * * p<0,01 ; * * * p<0,001$

werden jeweils die Werte des Gesamtmodells, in das (unter Berücksichtigung der Klassenstruktur und Kontrolle des Prätestwertes sowie des Geschlechts) die Einschätzung der Lernenden bzgl. der drei oben beschriebenen Qualitätskriterien gleichzeitig eingehen.

Deutlich wird, dass es Zusammenhänge mit dem von den Schüler*innen eingeschätzten Ausmaß der lernunterstützenden Rückmeldung auf beide motivationalen Aspekte gibt. Die Regressionsgewichte zeigen dabei, dass es sich durchaus um substanzielle Effekte handelt.

Etwas geringer sind die Zusammenhänge zur Skala passende und hilfreiche Ziele. Hypothesenkonform ist hier v. a. der Zusammenhang zur Anstrengungsbereitschaft. Wenn Lernende die vereinbarten Ziele als passend und hilfreich wahrnehmen, dann steigt ihre Bereitschaft, sich zu bemühen, diese auch zu erreichen.

Keine Zusammenhänge finden sich jedoch zur Skala „Berücksichtigung der Selbsteinschätzung“ - auch dann nicht, wenn dieser Wert ohne die beiden anderen Skalen zur Wahrnehmung der LEG in ein gesondertes Modell aufgenommen wird.

\section{Zusammenfassung und Diskussion}

Zusammenfassend kann festgehalten werden, dass die Befunde unsere zentrale Hypothese bestärken: Es gibt Zusammenhänge zwischen einer hohen Qualität der Lernentwicklungsgespräche (hier untersucht aus der Sicht der Lernenden) und der günstigen Entwicklung von Anstrengungsbereitschaft und schulischem Selbstkonzept. Dies gilt v.a. für die Wahrnehmung von lernunterstützender Rückmeldung sowie von passenden und hilfreichen Zielen. Keine Zusammenhänge lassen sich zur Berücksichtigung der eigenen Einschätzung nachweisen.

Diese Berücksichtigung der eigenen Einschätzung wird dabei - genauso wie das Ausmaß lernunterstützender Rückmeldungen sowie die Vereinbarung passender und hilfreicher Ziele - von den Lernenden im Mittel als relativ hoch eingeschätzt; diese Einschätzung und damit die wahrgenommene Umsetzung der Qualitätskriterien variiert jedoch auch zwischen einzelnen Klassen und einzelnen Kindern. Diese Varianz steht in Einklang mit den Ergebnissen von Beobachtungsdaten (Ertl et al. im Druck). Dort sind die Unterschiede noch deutlich markanter: ICC-Werte von bis zu 
0,64 verweisen auf deutliche Unterschiede in der Umsetzung einzelner Elemente zwischen den Klassen - zugleich zeigen sich deutliche Unterschiede zwischen den verschiedenen LEG einzelner Lehrpersonen. Da bezüglich der konkreten Umsetzung von LEG kaum Vorgaben bestehen und dadurch eine Vielzahl an Formen existiert, ist dies nicht besonders verwunderlich. Die Verbindung der beiden Datensätze steht jedoch noch aus: Auch wenn vermutet werden kann, dass sich die unterschiedlichen Einschätzungen der Lernenden durch die unterschiedliche Qualität der LEG erklären lassen, so ist jedoch in weiteren Analysen zu überprüfen, inwieweit Beobachtersicht und Einschätzung der Kinder übereinstimmen. Anschließend ist zu eruieren, inwieweit die hier gefundenen Zusammenhänge zwischen Qualität und Entwicklung motivationaler Aspekte des Lernens sich auch dann zeigen, wenn nicht die Einschätzung der Kinder, sondern die eingeschätzte Qualität der LEG aus Beobachtersicht berücksichtigt wird.

Unsere hier vorgestellten Ergebnisse reihen sich in die Befundlage zur Bedeutsamkeit von Tiefenstrukturen des Unterrichts auf das Lernen von Schüler*innen ein (z.B. Drechsel und Schindler 2019) und erweitern diese auf den Aspekt der Leistungsrückmeldung. Da die Einschätzung der Lernenden als Maß für die Qualität der LEG diente, stehen unsere Befunde zudem im Einklang zu Ergebnissen aus Studien zu Feedback. Auch dort zeigte sich, dass vor allem entscheidend ist, inwieweit die Lernenden selbst die erhaltene Rückmeldung als nützlich und hilfreich erachten (Harks et al. 2014).

Die Befunde sind aus unserer Sicht auch aus dem Grund bemerkenswert, da sich aufgrund einer vergleichsweise kleinen Intervention (betrachtet wurde die Varianz in einem maximal 30-minütigen Gespräch) Effekte auf recht grundlegende und stabile Konstrukte, wie z.B. die Anstrengungsbereitschaft und das schulische Selbstkonzept, nachweisen ließen. Dies deutet auf eine große subjektive Bedeutsamkeit der LEG für die Lernenden hin.

Nicht hypothesenkonform ist der Befund zur Berücksichtigung der Selbsteinschätzung der Schüler*innen im Gespräch. In keinem Modell zeigten sich überzufällige Zusammenhänge zur Entwicklung von Anstrengungsbereitschaft und Selbstkonzept. Ein möglicher Erklärungsansatz könnte sein, dass von den Lernenden an die LEG in Bayern keine zu großen Erwartungen bezüglich ihrer Selbstbestimmung gelegt werden, da die LEG vorrangig in ihrer Funktion als Ersatz des Zwischenzeugnisses gesehen werden. Von daher sind in diesem Setting die beiden anderen Qualitätsmerkmale von größerer Bedeutung. Untersuchungen in anderen Bundesländern oder Schulformen, in denen Eltern-Kind-Gespräche mit anderen Erwartungen geführt werden (z.B. Betz et al. 2019) könnten hier evtl. zur Klärung bzw. Ausdifferenzierung dieses Befunds beitragen. Eine weitere mögliche Erklärung ergibt sich zudem aus den Befunden von Bonanati (2018, S. $411 \mathrm{ff}$.). Sie konnte in ihren Gesprächsanalysen zeigen, dass durch die (steuernde) Gesprächsführung der Lehrpersonen die Schülerselbsteinschätzungen weniger dem konkreten Austausch über das Lernen dienen, sondern eher einer Einübung einer bestimmten Technik der Selbsteinschätzung. Sie kommt zu dem Schluss, dass sich die Lernenden dabei bemühen, sich als gute Schüler*innen darzustellen und ihre eigene Einschätzung der der Lehrperson anpassen. Damit hätten die Schüler*innen jedoch gar nicht das Ziel, ihre Selbsteinschätzung prominent in das Gespräch einzubringen oder gar gegenüber 
einer anderen Einschätzung zu verteidigen. Es ist geplant, diese Überlegung durch die Analyse der vorhandenen Videodaten zu überprüfen.

Inwiefern es sich bei den gefundenen Effekten um stabile Effekte handelt, ist in weiteren Studien zu untersuchen. Dennoch ist bereits jetzt ersichtlich, dass in der Umsetzung der LEG auf ein konsequentes lernförderliches Feedback und die Vereinbarung passender und hilfreicher Ziele zu achten ist, was bisher in der Umsetzung - sowohl aus Kindersicht als auch aus Beobachtersicht - teilweise deutlich zwischen einzelnen Klassen und einzelnen LEG variiert. Grundsätzlich ist dabei zu berücksichtigen, dass es nicht ausreicht, wenn lernförderliches Feedback ausschließlich in LEG gegeben wird. Eine Passung zum Unterrichtsgeschehen während des Schuljahres ist hier sicherlich anzustreben. Dies begründet sich auch aus Befunden, dass Lernende auf Feedback reagieren müssen, damit eine aktive Informationsverarbeitung in Gang gesetzt wird (Hattie 2015, S. 207 ff.). Damit dies gelingen kann, ist regelmäßig entsprechendes Feedback erforderlich. Auch sollten es die Lernenden gewohnt sein, mit konkreten, festgelegten Zielen zu arbeiten, damit entsprechende Lern- und Arbeitsprozesse initiiert werden (Grassinger et al. 2019, S. 222f.). Gleiches gilt für die Selbstreflexion und Selbstbewertung.

Schließlich ist zu eruieren, inwiefern die Qualität der LEG von professionellen Kompetenzen der Lehrpersonen beeinflusst wird, insbesondere durch das pädagogisch-psychologische Wissen und professionelle Überzeugungen. Da, wie oben erwähnt, die konkrete Umsetzung der LEG den Lehrkräften im Wesentlichen freigestellt ist, könnten diese Aspekte bedeutsam sein. Zu überprüfen ist dann auch, inwieweit über entsprechende Lehrerfortbildungen zu lernunterstützenden Rückmeldungen sowie zur Vereinbarung passender und hilfreicher Ziele die Qualität von LEG erhöht werden kann. Selbstverständlich sollte die qualitative Gestaltung von LEG aufgrund ihrer zunehmenden Bedeutung auch bereits in der Lehrerausbildung entsprechend berücksichtigt werden. Aufgrund der Bedeutung für die Weiterentwicklung motivationaler Aspekte des Lernens und den diagnostischen Aspekten der Leistungsbeurteilung bieten sich an dieser Stelle auch interdisziplinäre Ausbildungsformate an (Ertl et al. im Druck).

Förderung In diesem Beitrag wurde eine Teilfragestellung eines von der Deutschen Forschungsgemeinschaft geförderten Projekts (FKZ: DO 2184/1-1) bearbeitet.

Funding Open Access funding enabled and organized by Projekt DEAL.

Open Access Dieser Artikel wird unter der Creative Commons Namensnennung 4.0 International Lizenz veröffentlicht, welche die Nutzung, Vervielfältigung, Bearbeitung, Verbreitung und Wiedergabe in jeglichem Medium und Format erlaubt, sofern Sie den/die ursprünglichen Autor(en) und die Quelle ordnungsgemäß nennen, einen Link zur Creative Commons Lizenz beifügen und angeben, ob Änderungen vorgenommen wurden.

Die in diesem Artikel enthaltenen Bilder und sonstiges Drittmaterial unterliegen ebenfalls der genannten Creative Commons Lizenz, sofern sich aus der Abbildungslegende nichts anderes ergibt. Sofern das betreffende Material nicht unter der genannten Creative Commons Lizenz steht und die betreffende Handlung nicht nach gesetzlichen Vorschriften erlaubt ist, ist für die oben aufgeführten Weiterverwendungen des Materials die Einwilligung des jeweiligen Rechteinhabers einzuholen.

Weitere Details zur Lizenz entnehmen Sie bitte der Lizenzinformation auf http://creativecommons.org/ licenses/by/4.0/deed.de. 


\section{Literatur}

Andrade, H. L. (2010). Summing up and moving forward. Key challenges and future directions for research and development in formative assessment. In H.L. Andrade \& G. J. Cizek (Hrsg.), Handbook of formative assessment (S. 334-351). New York: Routledge.

Betz, T., Bischoff-Pabst, S., Eunicke, N., \& Menzel, B. (2019). Kinder zwischen Chancen und Barrieren. Zum Verhältnis von Schule und Familie aus der Sicht von Kindern: ihre Perspektiven, ihre Positionen. Forschungsbericht 2. Gütersloh: Bertelsmann Stiftung.

Black, P., \& Wiliam, D. (2009). Developing the theory of formative assessment. Educ Asse Eval Acc, 21, 5-31. https://doi.org/10.1007/s11092-008-9068-5.

Bonanati, M. (2018). Lernentwicklungsgespräche und Partizipation. Rekonstruktion zur Gesprächspraxis zwischen Lehrpersonen, Grundschülern und Eltern. Wiesbaden: Springer VS.

Brandstätter, V., \& Hennecke, M. (2018). Ziele. In J. Heckenhausen \& H. Heckenhausen (Hrsg.), Motivation und Handeln (4. Aufl. S. 331-353). Berlin: Springer.

Brunstein, J.C., \& Heckhausen, H. (2018). Leistungsmotivation. In J. Heckhausen \& H. Heckhausen (Hrsg.), Motivation und Handeln (4. Aufl. S. 163-221). Berlin, Heidelberg: Springer.

Dollinger, S. (2019). Lernunterstützende Rückmeldung in Lernentwicklungsgesprächen. Zeitschrift für Grundschulforschung, 12(1), 197-212. https://doi.org/10.1007/s42278-019-00040-z.

Dollinger, S. (2020). Lernentwicklungsgespräche - Umsetzung lernunterstützender Rückmeldung. In N. Skorsetz, M. Bonanati \& D. Kucharz (Hrsg.), Diversität und Ungleichheit. Herausforderungen an die Integrationsleitung der Grundschule (S. 154-158). Wiesbaden: Springer VS.

Dollinger, S., \& Hartinger, A. (2019). Lernentwicklungsgespräche - Praktische Umsetzung und Konsequenzen. Die Schulleitung, 46(4), 4-12.

Dollinger, S., Hartinger, A., \& Klippel, E. (2020a). Qualitätskriterien von Lernentwicklungsgesprächen. In S. Dollinger \& A. Hartinger (Hrsg.), Lernentwicklungsgespräche in der Grundschule. Erprobte Praxisbausteine (S. 17-28). Berlin: Cornelsen.

Dollinger, S., Hartinger, A., \& Klippel, E. (2020b). Theoretische Grundlagen: Das Lernentwicklungsgespräch. In S. Dollinger \& A. Hartinger (Hrsg.), Lernentwicklungsgespräche in der Grundschule. Erprobte Praxisbausteine (S. 9-10). Berlin: Cornelsen.

Drechsel, B., \& Schindler, A. (2019). Unterrichtsqualität. In D. Urhahne, M. Dresel \& F. Fischer (Hrsg.), Psychologie für den Lehrberuf (S. 353-372). Berlin: Springer.

Dresel, M., \& Lämmle, L. (2017). Motivation. In T. Götz (Hrsg.), Emotion, Motivation und selbstreguliertes Lernen (2. Aufl. S. 80-142). Paderborn: Schöningh.

Eccles, J., \& Wigfield, A. (2002). Motivational beliefs, values and goals. Annual Review of Psychology, $53,109-132$.

Ehm, J.-H., Hasselhorn, M., \& Schmiedek, F. (2019). Analyzing the developmental relation of academic self-concept and achievement in elementary school children: Alternative models point to different results. Developmental Psychology, 55, 2336-2351. https://doi.org/10.1037/dev0000796.

Ertl, S., Kücherer, B., \& Hartinger, A. (im Druck). Berücksichtigung individueller Lernprozesse in Lernentwicklungsgesprächen. In A. Hartinger, M. Dresel, E. Matthes, U. Nett \& K. Peuschel (Hrsg.), Lehrkräfteprofessionalität im Umgang mit Heterogenität - theoretische Konzepte, Förderansätze, empirische Befunde. Münster: Waxmann. im Druck.

Ertl, S., Kücherer, B., \& Hartinger, A. (in Vorbereitung). Vergleichsprozesse in Lernentwicklungsgesprächen

Furtak, E. M., Kiemer, K., Circi, R. K., Swanson, R., de León, V., Morrison, D., \& Heredia, S. C. (2016). Teachers' formative assessment abilities and their relationship to student learning: findings from a fouryear intervention study. Instructional Science, 44(3), 267-291.

Grassinger, R., Dickhäuser, O., \& Dresel, M. (2019). Motivation. In D. Urhahne, M. Dresel \& F. Fischer (Hrsg.), Psychologie für den Lehrberuf (S. 207-227). Berlin: Springer.

Grittner, F. (2015). Portfoliogespräche. In H. De Boer \& M. Bonanati (Hrsg.), Gespräche über Lernen Lernen im Gespräch (S. 161-176). Wiesbaden: Springer VS.

Häbig, J. (2018). Lernentwicklungsgespräche aus der Sicht von Schülerinnen und Schülern. Eine MixedMethods-Studie an Gymnasien. Wiesbaden: Springer VS.

Harks, B., Rakoczy, K., Klieme, E., Hattie, J., \& Besser, M. (2014). Indirekte und moderierte Effekte von schriftlicher Rückmeldung auf Leistung und Motivation. In H. Ditton \& A. Müller (Hrsg.), Feedback und Rückmeldungen. Theoretische Grundlagen, empirische Befunde, praktische Anwendungsfelder (S. 163-194). Münster: Waxmann. 
Hattie, J. (2009). Visible learning. A synthesis of over 800 meta-analyses relating to achievement. London: Routledge.

Hattie, J. (2015). Lernen sichtbar machen (3. Aufl.). Überarbeitete und deutschsprachige Ausgabe von „Visible Learning“" besorgt von W. Beywl \& K. Zierer. Hohengehren: Schneider.

Hattie, J., \& Timperley, H. (2007). The power of feedback. Review of Educational Research, 77, 81-112.

Helmke, A. (1998). Vom Optimisten zum Realisten? Zur Entwicklung des Fähigkeitsselbstkonzeptes vom Kindergarten bis zur 6. Klassenstufe. In F.E. Weinert (Hrsg.), Entwicklung im Kindesalter (S. 115-132). Weinheim: Beltz.

Ingenkamp, K.-H. (1971). Die Fragwürdigkeit der Zensurengebung. Weinheim: Beltz.

Kollar, I., \& Fischer, F. (2019). Lehren und Unterrichten. In D. Urhahne, M. Dresel \& F. Fischer (Hrsg.), Psychologie für den Lehrberuf (S. 333-351). Berlin: Springer.

Maier, U. (2010). Formative Assessment - Ein erfolgsversprechendes Konzept zur Reform von Unterricht und Leistungsmessung? Zeitschrift für Erziehungswissenschaft, 13, 293-308.

Marsh, H. W. (1986). Verbal and math self-concepts: an internal/external frame of reference model. American Educational Research Journal, 23, 129-149.

Möller, J., \& Trautwein, U. (2015). Selbstkonzept. In E. Wild \& J. Möller (Hrsg.), Pädagogische Psychologie (2. Aufl. S. 178-199). Berlin, Heidelberg: Springer.

Moschner, B., \& Dickhäuser, O. (2018). Selbstkonzept. In D. H. Rost, J. R. Sparfeldt \& S. R. Buch (Hrsg.), Handwörterbuch Pädagogische Psychologie (5. Aufl. S. 750-756). Weinheim, Basel: Beltz.

Müller, R. (2013). Bedingungsfaktoren schulischer Leistungen in der Grundschule - (Womit) Kann Schulerfolg prognostiziert werden? Eine Längsschnittuntersuchung an Grundschulen und Schulen der Sekundarstufe I in Bayern und Sachsen. München: Herbert Utz.

Mundwiler, V. (2017). Beurteilungsgespräche in der Schule. Eine gesprächsanalytische Studie zur Interaktion zwischen Lehrpersonen, Eltern sowie Schülerinnen und Schülern. Basler Studien zur deutschen Sprache + Literatur, Bd. 98. Tübingen: Narr/Francke/Attempto.

Munser-Kiefer, M., Martschinke, S., \& Hartinger, A. (2016). Selbstbestimmtes Lernen im jahrgangsgemischten Unterricht der 3. und 4. Klasse - erste Ergebnisse aus einer Evaluationsstudie. In F. Heinzel \& K. Koch (Hrsg.), Individualisierung im Grundschulunterricht: Anspruch, Realisierung und Risiken (S. 87-91). Wiesbaden: Springer VS.

Muthén, L. K., \& Muthén, B.O. (2017). Mplus user's guide (8. Aufl.). Los Angeles: Muthén \& Muthén.

Nett, U., \& Schilling, C. (2020). Vorbereitung der Lernentwicklungsgespräche. In S. Dollinger \& A. Hartinger (Hrsg.), Lernentwicklungsgespräche in der Grundschule. Erprobte Praxisbausteine (S. 40-70). Berlin: Cornelsen.

Poloczek, S., Karst, C., Praetorius, A.-K., \& Lipowsky, F. (2011). Generalisten oder Spezialisten? Bereichsspezifität und leistungsbezogene Zusammenhänge des schulischen Selbstkonzepts von Schulanfängern. Zeitschrift für Pädagogische Psychologie, 25(3), 173-183.

Praetorius, A.-K., Kastens, C., Hartig, J., \& Lipowsky, F. (2016). Haben Schüler mit optimistischen Selbsteinschätzungen die Nase vorn? Zeitschrift für Entwicklungspsychologie und Pädagogische Psychologie, 48(1), 14-26.

Rauer, W., \& Schuck, K. D. (2003). FEESS 3-4. Fragebogen zur Erfassung emotionaler und sozialer Schulerfahrungen von Grundschulkindern dritter und vierter Klassen. Göttingen: Beltz.

Rauer, W., \& Schuck, K. D. (2004). FEESS 1-2. Fragebogen zur Erfassung emotionaler und sozialer Schulerfahrungen von Grundschulkindern erster und zweiter Klassen. Göttingen: Beltz.

Renner, G., Martschinke, S., Munser-Kiefer, M., \& Steinmüller, S. (2011). Diagnose und Förderung des Selbstkonzepts im Anfangsunterricht. In F. Hellmich (Hrsg.), Selbstkonzepte im Grundschulalter. Modelle, empirische Ergebnisse, pädagogische Konsequenzen (S. 247-265). Stuttgart: Kohlhammer.

Rheinberg, F., \& Fries, S. (2018). Motivationstraining und Motivierung. In D. H. Rost, J.R. Sparfeldt \& S. R. Buch (Hrsg.), Handwörterbuch Pädagogische Psychologie (5. Aufl. S. 569-574). Weinheim, Basel: Beltz.

Rollet, B., \& Rollet, W. (2018). Anstrengungsvermeidung. In D.H. Rost, J.R. Sparfeldt \& S.R. Buch (Hrsg.), Handwörterbuch Pädagogische Psychologie (5. Aufl. S. 8-13). Weinheim, Basel: Beltz.

Sacher, W. (2014). Leistungen entwickeln, überprüfen und beurteilen. Bewährte und neue Wege für die Primar- und Sekundarstufe (6. Aufl.). Bad Heilbrunn: Klinkhardt.

Schmidinger, E., Hofmann, F., \& Stern, T. (2016). Leistungsbeurteilung unter Berücksichtigung ihrer formativen Funktion. In M. Bruneforth, F. Eder, K. Krainer, C. Schreiner, A. Seel \& C. Spiel (Hrsg.), Fokussierte Analysen bildungspolitischer Schwerpunktthemen. Nationaler Bildungsbericht Österreich 2015, (Bd. 2, S. 59-93). Graz: Leykam.

Schmude, K. (2002). Wie werden Berichtszeugnisse realisiert? In R. Valtin (Hrsg.), Was ist ein gutes Zeugnis? Noten und verbale Beurteilungen auf dem Prüfstand (S. 77-88). Weinheim, München: Juventa. 
Schunk, D. H. (1996). Goal and self-evaluative influences during children's cognitive skill learning. American Educational Research Journal, 33(2), 359-382.

Shavelson, R. J., Hubner, J. J., \& Stanton, G. C. (1976). Self-concept: validation of construct interpretations. Review of Educational Research, 46(3), 407-441.

Spinath, B. (2004). Determinanten von Fähigkeitsselbstwahrnehmungen im Grundschulalter. Zeitschrift für Entwicklungspsychologie und Pädagogische Psychologie, 36(2), 63-68.

Stubbe, T. C., Bos, W., \& Euen, T. (2012). Der Übergang von der Primar- in die Sekundarstufe. In W. Bos, I. Tarelli, A. Bremerich-Vos \& K. Schwippert (Hrsg.), IGLU 2011. Lesekompetenzen von Kindern in Deutschland im internationalen Vergleich (S. 209-226). Münster: Waxmann.

Valtin, R., \& Wagner, C. (2002). Wie wirken sich Notengebung und verbale Beurteilung auf die leistungsbezogene Persönlichkeitsentwicklung aus? In R. Valtin (Hrsg.), Was ist ein gutes Zeugnis? Noten und verbale Beurteilungen auf dem Prüfstand (S. 113-137). Weinheim, München: Juventa.

Wilhelm, M. (2015). Dokumentiertes Lernentwicklungsgespräch. Eine Alternative zum Zwischenzeugnis an bayerischen Grundschulen. Schulverwaltung Bayern, 38(1), 4-10.

Wolff, F., Helm, F., Zimmermann, F., Nagy, G., \& Möller, J. (2018). On the effects of social, temporal, and dimensional comparisons on academic self-concept. Journal of Educational Psychology, 110(7), 1005-1025. https://doi.org/10.1037/edu0000248.

Wolff, F., Nagy, G., Retelsdorf, J., Helm, F., Köller, O., \& Möller, J. (2019). The 2I/E model: Integrating temporal comparisons into the internal/external frame of reference model. Journal of Educational Psychology, 111(7), 1131-1161. https://doi.org/10.1037/edu0000319.

Worbach, M., Drechsel, B., \& Carstensen, C.H. (2019). Messen und Bewerten von Lernergebnissen. In D. Urhahne, M. Dresel \& F. Fischer (Hrsg.), Psychologie für den Lehrberuf (S. 493-516). Berlin: Springer. 